\title{
Some Measures for COVID-19 Including Deep Ultraviolet Light-Emitting Diode (DUV-LED), Gc protein-derived Macrophage-Activating Factor (Gcmaf), and 5-Aminolevulinic Acid (5-ALA)
}

\author{
Hiroshi Bando ${ }^{1,2^{*}}$ \\ ${ }^{1}$ Medical Research/Tokushima University, Tokushima, Japan \\ ${ }^{2}$ Integrative Medicine Japan (IMJ), Shikoku Island Division, Tokushima, Japan
}

Corresponding Author: Hiroshi BANDO, MD, PhD, FACP ${ }^{\text {ORCID iD }}$

Address: Tokushima University /Medical Research, Nakashowa 1-61, Tokushima 770-0943, Japan. Tel: +81-90-31872485. Email:pianomed@bronze.ocn.ne.jp

Received date: 25 May 2021; Accepted date: 23 June 2021; Published date: 30 June 2021

Citation: Bando H. Some Measures for COVID-19 Including Deep Ultraviolet Light-Emitting Diode (DUV-LED), Gc protein-derived Macrophage-Activating Factor (Gcmaf), and 5-Aminolevulinic Acid (5-ALA). Asp Biomed Clin Case Rep. 2021 Jun 30;4(2):110-13.

Copyright (C) 2021 Bando H. This is an open-access article distributed under the Creative Commons Attribution License, which permits unrestricted use, distribution, and reproduction in any medium provided the original work is properly cited.

\begin{abstract}
Several trials for the COVID-19 pandemic are found. Prof. Shunji Nakamura, Tokushima University was awarded the 2014 Nobel prize in Physics for light-emitting diodes (LEDs). Deep ultraviolet LED (DUV-LED) causes SARS-CoV-2 inactivation as $99.9 \%$ by $265 \mathrm{~nm}$. Gc protein-derived macrophage-activating factor (GcMAF)-based immunotherapy has a wider application for serious infection, chronic fatigue syndrome, and cancers. Covid center in Naples submitted a phase II report concerning GcMAF and COVID-19 in Feb 2021. 5aminolevulinic acid (5-ALA) and its metabolite protoporphyrin IX (PpIX) show anti-inflammatory and antiviral effects for Zika, Dengue, influenza A viruses, and SARS-CoV-2. These results hopefully contribute to the research development for COVID-19.
\end{abstract}

\section{Keywords}

Light Emitting Diode, Deep Ultraviolet Light Emitting Diode, Gc protein-derived Macrophage-Activating Factor, 5-Aminolevulinic Acid, Protoporphyrin IX, Severe Acute Respiratory Syndrome Coronavirus-2

\section{Abbreviations}

LED: Light Emitting Diode; DUV-LED: Deep Ultraviolet Light Emitting Diode; Gcmaf: Gc protein-derived Macrophage-Activating Factor; 5-ALA: 5-Aminolevulinic Acid; PPIX: Protoporphyrin IX; SARS-Cov-2: Severe Acute Respiratory Syndrome Coronavirus-2

Across the world, the COVID-19 pandemic has been a medical, social, human, and economic crucial problem. A variety of actual management for daily life and novel research for treatments have been continued [1]. As a matter of fact, the excess mortality of COVID19 in Japan has been the lowest with minus 15000 in the world [2-4]. Among them, several new trials have been found in Japan, which are introduced and described in this article.

Formerly, a historical invention of white InGaN based light emitting diodes (LEDs) and blue laser 
Citation: Bando H. Some Measures for COVID-19 Including Deep Ultraviolet Light-Emitting Diode (DUV-LED), Gc protein-derived Macrophage-Activating Factor (Gcmaf), and 5-Aminolevulinic Acid (5-ALA). Asp Biomed Clin Case Rep. 2021 Jun 30;4(2):110-13.

diodes (LDs) was achieved by Prof. Shunji Nakamura, Tokushima University. He was awarded the 2014 Nobel prize in Physics [5]. Using this deep ultraviolet light-emitting diode (DUV-LED), quantitative evaluation of severe acute respiratory syndrome coronavirus 2 (SARS-CoV-2) inactivation was reported as $99.9 \%$ in 2021 [6]. For the achievement of this level, total doses of $1.8 \mathrm{~mJ} / \mathrm{cm}^{2}$ for $265 \mathrm{~nm}, 3.0 \mathrm{~mJ} / \mathrm{cm}^{2}$ for $280 \mathrm{~nm}$, and $23 \mathrm{~mJ} / \mathrm{cm}^{2}$ for $300 \mathrm{~nm}$ are required. These data could provide the quantitative antiviral clinical efficacy by DUV irradiation on SARS-COV-2, which serves the fundamental knowledge of inactivation methods for SARS-COV-2. This project was supported by Tokushima Prefectural Government and Tokushima University. According to the data mentioned above, some experiments were conducted using LEDs systems by changing the condition [7]. As a result, a wavelength of $260 \mathrm{~nm}$ was most effective for the inactivation of coronavirus particles. This research will be expected to develop for broad application worldwide.

Another international research has been found. Clinical research for Gc protein-derived macrophageactivating factor (GcMAF) has been continued in Tokushima University for years [8]. GcMAF has a variety of functions as an immune modulator, including macrophage activation, anti-tumor activity, and anti-angiogenic activity. Consequently, GcMAFbased immunotherapy shows a wider application for serious infection, chronic fatigue syndrome, multiple sclerosis, and several cancers [9]. GcMAF has the possibility of the role for suppressing the severity of COVID-19-induced immune responses [10]. Covid center in Naples, Italy summarized the phase II report concerning GcMAF and COVID-19 pneumonia with 104 references and 63 pages in Feb 2021 [11]. Further development for the interaction of GcMAF and COVID19 will be expected.

The third topic is 5-aminolevulinic acid (5-ALA). The research group of Tokushima University formerly reported the involvement of 5-ALA, which is the precursor of protoporphyrin IX (PpIX) for the possible sonosensitizers for sonodynamic treatment of cancers [12]. 5-ALA is a synthesized amino acid and has been applied for various purposes such as anticancer therapy or as a dietary supplement. Recently, it has been showed potently inhibition of infection of SARSCoV-2 in cell culture. The detailed research has been continued at Nagasaki University in Japan [13]. Its antiviral efficacy was detected in both human and nonhuman cells with no significant cytotoxicity.

5-ALA is one of the natural amino acids and exists in plants, bacteria, fungi, and animals. The conjugation of 8 molecules of 5-ALA bring the production of PPIX, which can generate heme with the ferrous ion insertion. Since 5-ALA strengthens the aerobic energy metabolism, it is applied for improved metabolism in such as diabetes mellitus [14]. Its application was broadened for infections such as malaria [15]. Furthermore, PPIX was known to show antiviral efficacy for various viruses including Zika virus, Dengue virus, influenza A virus, and also SARS-CoV-2 [16].

It has been reported that exogenously supplied 5ALA affects the host through various mechanisms [17]. Metabolites of 5-ALA such as PPIX and hem seem to affect viral infection existing inside the host cells. A recent report demonstrated that a G-quadruplex ( $\left.\mathrm{G}_{4}\right)$ binding compound could inhibit SARS-CoV-2 replication [18]. G4s show tetra-helical structures made of guanine-rich regions of DNA or RNA, which can regulate gene expression, genome stability, and adequate control of protein quality [19]. G4 structures have been observed in the genome of many kinds of viruses such as coronaviruses and can regulate viral replication cycles. As to recent studies, $\mathrm{G}_{4}$ structures of the SARS-CoV-2 RNA genome and its interaction with the binding proteins would become possibly antiviral targets to attach COVID-19 [18,20]. In addition, heme is the metabolites of 5-ALA and a ligand of $\mathrm{G}_{4}$ structures [21]. Consequently, exogenous 5-ALA administration leads to increased production of PPIX and heme inside the host cells, which may interfere with the interaction of $\mathrm{G}_{4}$ structures or virus genome leading to inhibition of SARS-CoV-2 infection.

5-ALA is synthesized in most plants and animals, and then we always consume it within our daily foods [22]. Then, it is safely provided to humans as a supplement or medicine for a wider population 
Citation: Bando H. Some Measures for COVID-19 Including Deep Ultraviolet Light-Emitting Diode (DUV-LED), Gc protein-derived Macrophage-Activating Factor (Gcmaf), and 5-Aminolevulinic Acid (5-ALA). Asp Biomed Clin Case Rep. 2021 Jun 30;4(2):110-13.

including mild cases of patients with COVID-19 [23]. Moreover, 5-ALA shows anti-inflammatory efficacy in humans, and then it is possible to provide rather severe cases for the combined therapy for antiinflammation and antiviral activity [22]. Concerning the research of 5-ALA and COVID-19, experiments using swine have been found [24,25]. Consequently, further studies of antiviral function by animal experiments and mechanistic research will be expected.

As mentioned above, several recent topics were introduced and discussed in this article. They include i) SARS-CoV-2 inactivation was $99.9 \%$ by DUV-LED, ii) GcMAF would be effective as phase II report from Italy, iii) 5-ALA would be applied for COVID-19. The information will be hopefully prevalent across the world rapidly, leading to the health and happiness of all people with the development of research of COVID-19.

\section{Conflict of Interest}

The author has read and approved the final version of the manuscript. The author has no conflicts of interest to declare.

\section{References}

[1] Ministry of Health and Welfare Japan. Guideline of treatment for COVID-19 $5^{\text {th }}$ Edition. 2021 May 26;1-59. Available from:

https://www.mhlw.go.jp/content/ooo785119.pdf

[2] Bando H. Current Situation on the Excess Mortality of COVID-19 for Various Countries. Global J Infect Dis Immune Ther 2021 May 27; 3(1): 112-14

[3] Karlinsky A, Kobak D. The World Mortality Dataset: Tracking excess mortality across countries during the COVID-19 pandemic. medRxiv [Preprint]. 2021 Jan 29:2021.01.27.21250604. [PMID: 33532789]

[4] Bando H. Fewer Annual Deaths in Japan with Negative Excess Mortality for COVID-19. Asp Biomed Clin Case Rep. 2021 Apr 06;4(1):84-87.

[5] Nakamura S. Background Story of the Invention of Efficient InGaN Blue-Light-Emitting Diodes (Nobel Lecture). Angew Chem Int Ed Engl. 2015 Jun 26;54(27):7770-88. [PMID: 26031499]

[6] Minamikawa T, Koma T, Suzuki A, Mizuno T, Nagamatsu K, Arimochi H, Tsuchiya K, Matsuoka K,
Yasui T, Yasutomo K, Nomaguchi M. Quantitative evaluation of SARS-CoV-2 inactivation using a deep ultraviolet light-emitting diode. Scientific Reports. 2021 Mar 3;11(1):1-9.

[7] Hovorov P, Kindinova A. APPLICATION OF LEDS IN DISINFECTION TECHNOLOGIES. InterConf. 2021 Apr 23; 50:517-20

[8] Inui T, Kubo K, Kuchiike D, Uto Y, Nishikata T, Sakamoto N, Mette M. Oral Colostrum Macrophageactivating Factor for Serious Infection and Chronic Fatigue Syndrome: Three Case Reports. Anticancer Res. 2015 Aug;35(8):4545-49. [PMID: 26168499]

[9] Inui $\mathrm{T}$, Amitani $\mathrm{H}$, Kubo K, Kuchiike D, Uto $\mathrm{Y}$, Nishikata T, Mette M. Case Report: A Non-small Cell Lung Cancer Patient Treated with GcMAF, Sonodynamic Therapy and Tumor Treating Fields. Anticancer Res. 2016 Jul;36(7):3767-70. [PMID: 27354652]

[10] Spadera L, Spadera M. Potential role of GcMAF in suppressing the severity of COVID-19-induced immune responses: Lesson learned from HIV. Med Hypotheses. 2020 Nov;144:110293. [PMID: 33254486]

[11] Naples ID, Ospedale del Mare AS, Naples C, Grasso E. Phase II clinical trial evaluating efficacy and safety of Oral immunotherapy with Third Generation Gc Protein derived Macrophage Activating Factor (GcMAF) in hospitalized patients with COVID-19 pneumonia. 23 Feb 2021. Study code: COral-MAF1. Available from:

https://www.clinicaltrials.gov/ProvidedDocs/71/NCTo 4845971/Prot_SAP_ICF_oo1.pdf

[12] Uto Y, Tamatani D, Mizuki Y, Endo Y, Nakanishi I, Ohkubo K, Fukuzumi S, Ishizuka M, Tanaka T, Kuchiike D, Kubo K, Inui T, Hori H. Evaluation of the sonosensitizing activities of 5-aminolevulinic acid and Sn(IV) chlorin e6 in tumor-bearing chick embryos. Anticancer Res. 2014 Aug;34(8):4583-87. [PMID: 25075103]

[13] Sakurai Y, Ngwe Tun MM, Kurosaki Y, Sakura T, Inaoka DK, Fujine K, Kita K, Morita K, Yasuda J. 5amino levulinic acid inhibits SARS-CoV-2 infection in vitro. Biochem Biophys Res Commun. 2021 Mar 19;545:203-207. [PMID: 33571909]

[14] Rehani PR, Iftikhar H, Nakajima M, Tanaka T, Jabbar Z, Rehani RN. Safety and Mode of Action of Diabetes Medications in comparison with 5Aminolevulinic Acid (5-ALA). J Diabetes Res. 2019 Nov 
Citation: Bando H. Some Measures for COVID-19 Including Deep Ultraviolet Light-Emitting Diode (DUV-LED), Gc protein-derived Macrophage-Activating Factor (Gcmaf), and 5-Aminolevulinic Acid (5-ALA). Asp Biomed Clin Case Rep. 2021 Jun 30;4(2):110-13.

6;2019:4267357. [PMID: 31781665]

[15] Suzuki S, Hikosaka K, Balogun EO, Komatsuya K, Niikura M, Kobayashi F, Takahashi K, Tanaka T, Nakajima M, Kita K. In vivo curative and protective potential of orally administered 5-aminolevulinic acid plus ferrous ion against malaria. Antimicrob Agents Chemother. 2015 Nov;59(11):696o-67. [PMID: 26324278]

[16] Gu C, Wu Y, Guo H, Zhu Y, Xu W, Wang Y, Zhou Y, Sun Z, Cai X, Li Y, Liu J, Huang Z, Yuan Z, Zhang R, Deng Q, Qu D, Xie Y. Protoporphyrin IX and verteporfin potently inhibit SARS-CoV-2 infection in vitro and in a mouse model expressing human ACE2. Sci Bull (Beijing). 2021 May 15;66(9):925-36. [PMID: 33318880]

[17] Ota U, Sugihara H, Abe F. 5-Aminolevulinic Acid (5-ALA): a precursor of heme: Fermentation, metabolism and usage. ALA-porphyrin science. 2013;2(1):3-17.

[18] Zhao C, Qin G, Niu J, Wang Z, Wang C, Ren J, Qu X. Targeting RNA G-Quadruplex in SARS-CoV-2: A Promising Therapeutic Target for COVID-19? Angew Chem Int Ed Engl. 2021 Jan 4;60(1):432-38. [PMID: 32939952]

[19] Varshney D, Spiegel J, Zyner K, Tannahill D, Balasubramanian S. The regulation and functions of DNA and RNA G-quadruplexes. Nat Rev Mol Cell Biol. 2020 Aug;21(8):459-74. [PMID: 32313204]

[20] Zhang R, Xiao K, Gu Y, Liu H, Sun X. Whole Genome Identification of Potential G-Quadruplexes and Analysis of the G-Quadruplex Binding Domain for SARS-CoV-2. Front Genet. 2020 Nov 27;11:587829. [PMID: 33329730]

[21] Lat PK, Liu K, Kumar DN, Wong KKL, Verheyen EM, Sen D. High specificity and tight spatial restriction of self-biotinylation by DNA and RNA G-Quadruplexes complexed in vitro and in vivo with Heme. Nucleic Acids Res. 2020 Jun 4;48(10):5254-67. [PMID: 32329781]

[22] Ito H, Nishio Y, Hara T, Sugihara H, Tanaka T, Li XK. Oral administration of 5-aminolevulinic acid induces heme oxygenase-1 expression in peripheral blood mononuclear cells of healthy human subjects in combination with ferrous iron. Eur J Pharmacol. 2018 Aug 15;833:25-33. [PMID: 29753693]

[23] Nakajima M, Kaketani K. Safety, Tolerability, and Efficacy of 5-aminolevulinic Acid Phosphate, an Inducer of Heme Oxygenase 1, in Combination with Sodium Ferrous Citrate for the Treatment of COVID-19 Patients. OSF Preprints; 2021. Available from: osf.io/azxsm

[24] Kobayashi E. Testing of human equivalent dose of health food 5-aminolevulinic acid using the experimental pig. Biomed Pharmacother. 2021 Jul;139:111629. [PMID: 33906o8o]

[25] Pickering BS, Smith G, Pinette MM, Embury-Hyatt C, Moffat E, Marszal P, Lewis CE. Susceptibility of Domestic Swine to Experimental Infection with Severe Acute Respiratory Syndrome Coronavirus 2. Emerg Infect Dis. 2021 Jan;27(1):104-12. [PMID: 33350904]

Keywords: Light Emitting Diode, Deep Ultraviolet Light Emitting Diode, Gc protein-derived Macrophage-Activating Factor, 5-Aminolevulinic Acid, Protoporphyrin IX, Severe Acute Respiratory Syndrome Coronavirus-2 